\title{
Musculoskeletal trauma in paediatric practice: the experience from Marseille
}

\author{
Philippe Petit
}

(C) Springer-Verlag 2011

Imaging technology has progressed dramatically in the last 30 years. Flat panel high-resolution imaging, ultra-thin computed tomography (CT) sections with two- and threedimensional reformatting, and high-contrast and -resolution magnetic resonance imaging (MRI) with dedicated coils have changed our confidence in the exploration of bone trauma. CT and MRI have definitively increased our diagnostic abilities, particularly in children following polytrauma. However, polytrauma only accounts for a minority of trauma cases admitted to an emergency department. This presentation will instead focus on the daily reality.

In a previous study performed over a 24-week period, we performed radiography in 3,128 anatomical locations in children admitted following trauma. Only $22 \%$ of the radiographic examinations were considered abnormal. In descending order, the hand and fingers, ankle, wrist, knee, elbow, foot and toes, and forearm were the most frequently examined locations. The rate of abnormal findings was $25.7 \%$ for the hand and fingers, $9.0 \%$ for the ankle, $42.5 \%$ for the wrist, $9.5 \%$ for the knee, $33.3 \%$ for the elbow, $18.3 \%$ for the foot and $43.2 \%$ for the forearm. When only the direct observation of a fracture was taken into account, these rates decreased for the ankle and knee to $2.6 \%$ and $1.9 \%$, respectively. Over the past ten years, these two joints in particular have been the focus of our attention.

Disclaimer Dr. Philippe Petit has no financial interests, investigational or off-label uses to disclose.

P. Petit $(\square)$

Department of Pediatric Radiology, Hôpital Timone Enfant,

264 rue Saint Pierre,

13385 Marseille Cedex 5, France

e-mail: ppetit@ap-hm.fr
Despite convincing data on the validity of the Ottawa Ankle Rules, referrals for ankle radiographs remain numerous. Our main explanation for this is the rotation in the emergency department of trainee doctors with various professional experiences and the fear of medicolegal proceedings. We have tried unsuccessfully in several projects to curtail these referrals. We have also investigated the underlying abnormality in swollen ankles when there was suspicion of sprain. Interestingly, in a series of 102 post-trauma MRI scans of ankles without radiographic fractures, we found only two ruptures of the lateral ligament in children with an open physis. Even more interesting, none of these had a classical Salter-Harris type I fracture of the distal fibula, which we believe is overdiagnosed. Two osteochondral avulsion fractures of the distal fibula, and microfractures of the foot were missed on radiographs. MRI has also been reported to be of high interest to modify the Salter-Harris classification of ankle fractures diagnosed on radiographs. However, our experience does not suggest this. In a series of 29 fractures, one patient was misclassified as Salter-Harris type III. MRI modified this classification to a Salter-Harris type III/Ogden type VI, but none of our patients had their therapeutic management changed following MRI. The point is that MRI of the ankle may show numerous abnormalities that are without clinical relevance. A delayed clinical examination, after three to four days' immobilisation, may also obviate numerous unnecessary imaging procedures. Ultrasounds may have a place in imaging after this period of short immobilization to detect the rare case of rupture of the talofibular and calcaneofibular ligaments.

However, expectancy is not suggested in knee trauma, where radiographs underestimate the severity of injury. CT or, even better, MRI must be performed as soon as a SalterHarris fracture is seen radiographically, or when there is 
discrepancy between clinical and radiographic findings. MRI is also mandatory when a Salter-Harris type I fracture is suspected in the proximal femoral physis (slipped proximal femoral epiphysis), despite negative radiographs. Bone marrow oedema on one or both sides of the physis confirms the clinical suspicion, and correct diagnosis allows timely orthopaedic management, decreasing the risk of avascular necrosis.

Traumatic pelvic fractures are rare in paediatric practice if we exclude polytrauma and osteochondral fractures. MRI may be confusing if there is extensive bone marrow and soft tissue oedema. Such findings may be of non-therapeutic interest, and may lead to overdiagnosis of bone or soft tissue tumours. CT may be of great help in identifying subtle osteochondral fractures and/or sign of myositis ossificans.

Minor spine trauma with normal radiographs may necessitate MRI, particularly if pain does not resolve within a few days. In this setting, STIR sequences in the sagittal and coronal planes allow diagnosis of trabecular bone contusions, and localise the site and extent of these. This helps the fitting of a proper analgesic cast.

Increasing pressure is put on the radiologist when trauma is sustained in children and adolescents who practice sport at a competitive level. Parents, managers and clubs expect fast high-tech imaging. We had the opportunity to prospectively investigate 26 young asymptomatic gymnasts on the French national team. Interestingly, in 34 elbows examined by MRI, two had lesions: one osteochondritis dissecans of the lateral condyle and one of the radial head. These findings did, however, not impact the training of these young athletes.

For chance the SFIPP (Société Francophoned'Imagerie Pédiatrique et Prénatale) was very reactive and convincing to allow to modify an act of the French Parliament, according to which, children, symptomatic or not, practicing various sports are expected to annually produce normal radiographs of the spine to be/remain enrolled by their respective sports committees. This official decree has been hopefully changed so that such abusive exposures can be curtailed. A more sensible strategy is now defined which included the need to perform spinal MRI in children at risk of acquired cervical spinal stenosis, e.g. those competing in rugby, diving and American football.

In conclusion, an imaging approach blind to the clinical context makes no sense. All needs for imaging explorations must be guided by the clinical findings and especially by the potential therapeutic consequences of abnormal imaging findings. Besides the obvious radiation risk and the implication for patients' quality of life, the approach also has significant financial impact. The annual cost of caring for children with traumatic injuries in the United States is estimated at $\$ 347$ billion. We suspect that this is partly due to unjustified imaging. A scientific definition of what is the best and potentially the only radiological exam to answer specific questions has to be found. Each traumatic location is specific and therefore needs a dedicated clinical and radiological strategy. 